\title{
Can complexity decreases in congestive heart failure?
}

\begin{abstract}
The complexity of a signal can be measured by the Recurrence period density entropy (RPDE) from the reconstructed phase space. We have chosen a window based RPDE method for the classification of signals, as RPDE is an average entropic measure of the whole phase space. We have observed the changes in the complexity in cardiac signals of normal healthy person (NHP) and congestive heart failure patients (CHFP). The results show that the cardiac dynamics of a healthy subject is more complex and random compare to the same for a heart failure patient, whose dynamics is more deterministic. We have constructed a general threshold to distinguish the border line between a healthy and a congestive heart failure dynamics. The results may be useful for wide range for physiological and biomedical analysis.
\end{abstract}

Keyword: Recurrence period density entropy; Complexity; Chaotic phenomeno; Cardiac signal; Deterministic; Stochastic dynamics 